\title{
Presumed Sertraline-Associated Maculopathy: A Case Series
}

\author{
Hedayat Javidi $\cdot$ Sarah Ah-Moye $\cdot$ Charles Hennings $\cdot$ Hemal Mehta $\cdot$ \\ Sajjad Mahmood (1)
}

Received: January 10, 2021 / Accepted: February 27, 2021 / Published online: March 20, 2021

(c) The Author(s) 2021

\begin{abstract}
Purpose: To report findings of maculopathy after treatment with sertraline in three patients. Methods: This case series includes three patients who presented with reduced visual acuity after treatment with sertraline for various psychiatric indications. All patients had been treated with sertraline for varying periods of time between 4 weeks and 5 years.

Results: Data were collected from three patients (age range, 27-68 years). All three patients were white females, with both eyes being affected in all cases. The range of presenting visual acuities was between 20/30 and 20/100 after presentation with central visual disturbance. All patients underwent comprehensive ocular examination and imaging with
\end{abstract}

H. Javidi · S. Mahmood ( $₫)$

Manchester Royal Eye Hospital, Manchester

University Hospitals NHS Foundation Trust,

Manchester, UK

e-mail: Sajjad.Mahmood@manchester.ac.uk

S. Ah-Moye $\cdot$ C. Hennings $\cdot$ H. Mehta

Royal Free London NHS Foundation Trust, London, UK

\section{H. Mehta}

Macular Research Group, Save Sight Institute,

Sydney University, Sidney, Australia

\section{S. Mahmood}

School of Health Sciences, University of

Manchester, Manchester, UK the main ocular findings being outer retinal layer and retinal pigment epithelial disruption. The follow-up period was between 1 and 9 months with final visual acuities between 20/25 and 20/100. Patients showed objective clinical evidence of phenotypically similar maculopathy supported by appropriate imaging Conclusion: In this cohort, we report the possible association of sertraline use and associated maculopathy in three patients. This is potentially significant, given the large numbers of patients treated with sertraline currently; however, further evidence is required to both quantify how common this association is and establish a possible causative mechanism.

Keywords: Maculopathy; OCT; Retina; Sertraline

\section{Key Summary Points}

In this case series, three patients treated with sertraline presented with reduced visual acuity. All patients had been treated with sertraline and developed a maculopathy after use.

These cases suggest the possibility of a rare association between sertraline use and a presumed sertraline-associated maculopathy. 


\section{DIGITAL FEATURES}

This article is published with digital features, including list digital features available e.g. a summary slide and video abstract, to facilitate understanding of the article. To view digital features for this article go to https://doi.org/10. 6084/m9.figshare.14125514.

\section{INTRODUCTION}

Selective serotonin reuptake inhibitors (SSRIs) are a drug class commonly used in the management of a wide range of psychiatric illnesses including major depressive disorder and generalised anxiety disorder [1]. Sertraline is one of the most commonly prescribed SSRIs, and was dispensed over 11 million times in the United Kingdom in 2016 alone [2]. Known common adverse effects of sertraline include gastrointestinal upset, insomnia, fatigue and headache [3].

Despite the widespread use of sertraline, few ocular side-effects have been documented, with some cases of optic neuropathy and acute angleclosure glaucoma reported [4, 5]. Additionally, five isolated, published cases of presumed sertraline-associated maculopathy have been described [6-10]. In this case series, we describe three further cases of presumed sertraline-induced maculopathy, to add to the growing body of evidence to support this rare association. Patient consent is on file.

Written informed consent for publication was obtained in two out of three cases. In the third case, the patient is not identifiable from the available information and has been included due to the rarity of this disease presentation.

\section{CASE PRESENTATION}

\section{Case 1}

A 36-year-old White woman presented with symptomatic central vision loss, followed by headaches. Initial visual acuity (VA) was 20/110 in the right eye (OD) and 20/100 in the left eye
(OS) (correcting with refraction to 20/85 OD and $20 / 80$ OS). On examination, the anterior and posterior segments were unremarkable, with no signs of intraocular inflammation, and normal intraocular pressures. The patient underwent magnetic resonance imaging (MRI) of the brain, lumbar puncture, cerebrospinal fluid (CSF) analysis and retinal nerve fibre layer (RNFL) analysis, all of which were unremarkable. RNFL analysis was performed using the Heidelberg Retina Tomograph (Heidelberg Engineering, Heidelberg, Germany). Stereopsis was normal. Visually evoked response was within normal limits. Optical coherence tomography (OCT) imaging demonstrated disruption of the outer retinal layers and retinal pigment epithelium (RPE) at the foveal centre (Fig. 1). OCT images were obtained using a Heidelberg Spectralis instrument.

The patient had a background of anxiety, depression, mood swings and hypertension. Her depression had been managed with sertraline $50 \mathrm{mg}$ once daily for over 5 years prior to onset of visual symptoms. At the time of presentation she was on no other medication of note in this context. She was advised to discontinue her sertraline, and at 6 months review was showing some architectural improvement on her OCT scan but no subjective or objective improvement in her visual acuity which was measured as $20 / 110$ bilaterally.

\section{Case 2}

A 27-year-old White woman presented with a 2-week history of bilateral, central visual disturbance. These symptoms had started 4 weeks after commencing sertraline $50 \mathrm{mg}$ once daily; the patient developed difficulty focusing and light sensitivity. BCVA was 20/100 bilaterally and Humphrey visual field testing (using the Humphrey Visual Field Analyzer 24-2 SITA Standard) demonstrated two central scotomas just above the fixation point, on either side of the vertical midline. Anterior segment examination was unremarkable, with no signs of intraocular inflammation, no vitreous activity and normal intraocular pressures. OCT imaging 


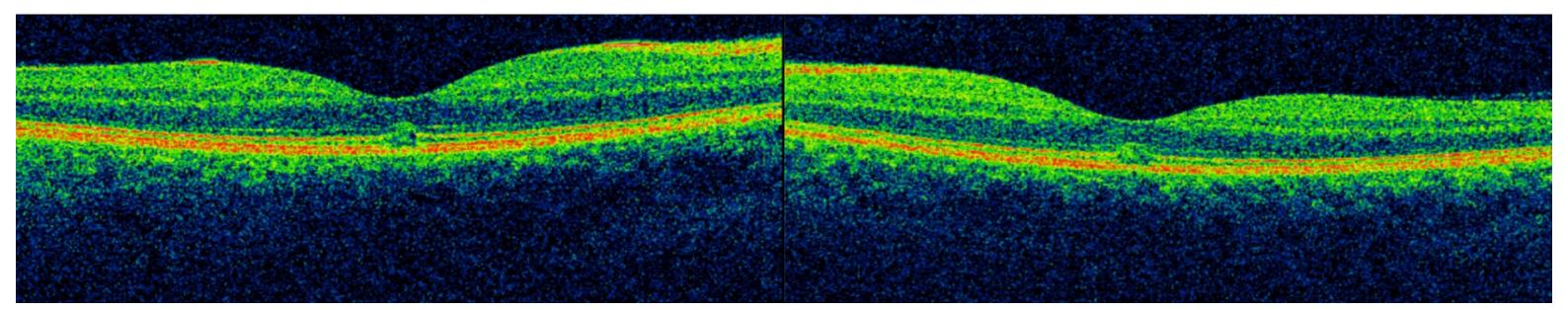

Fig. 1 Case 1 OCT scans of right and left eyes, respectively, taken on presentation, demonstrating symmetrical bilateral $\mathrm{RPE}$ and outer retinal disruption

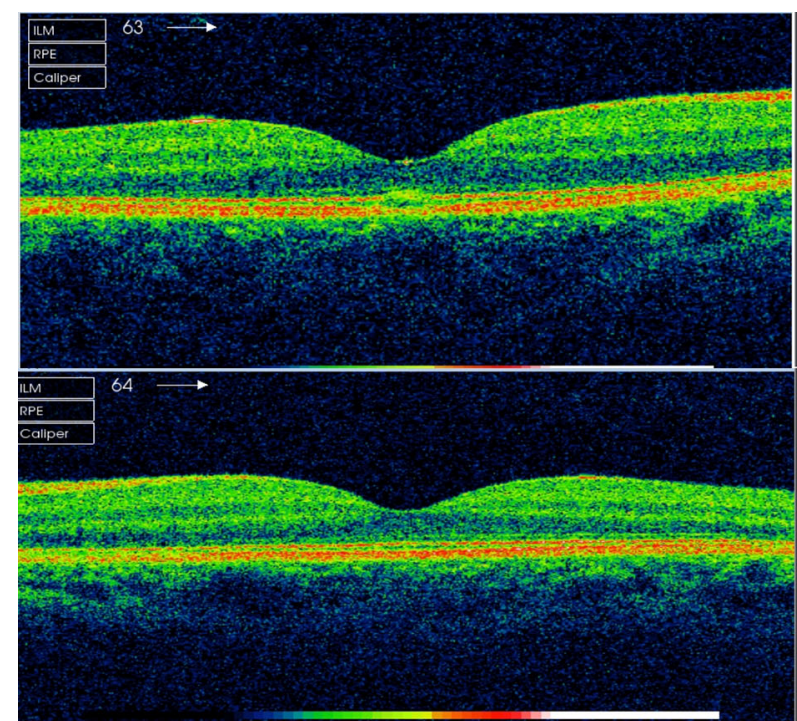

Fig. 2 Case 2 OCT OD and OS shown on the top row, respectively, at presentation, demonstrating bilateral RPE and outer retinal disruption on presentation. OCT OD and OS shown on the bottom row, respectively, 12 weeks

(Fig. 2) showed symmetrical disruption of the photoreceptor layer directly at the foveal centre.

The patient was already on treatment for panic attacks with propranolol $20 \mathrm{mg}$ once daily for 12 years prior to presentation; otherwise past medical history was unremarkable. She had a 15-units-per-week alcohol history but denied smoking or recreational drug use. The patient immediately ceased sertraline treatment. At 4-week follow-up, VA had improved to 20/25 bilaterally and ocular examination was entirely unremarkable, with healthy optic discs, maculae and peripheral retina. Previous foveal abnormalities noted on OCT had resolved (Fig. 2), with no evidence of disc swelling or nerve fibre layer loss.

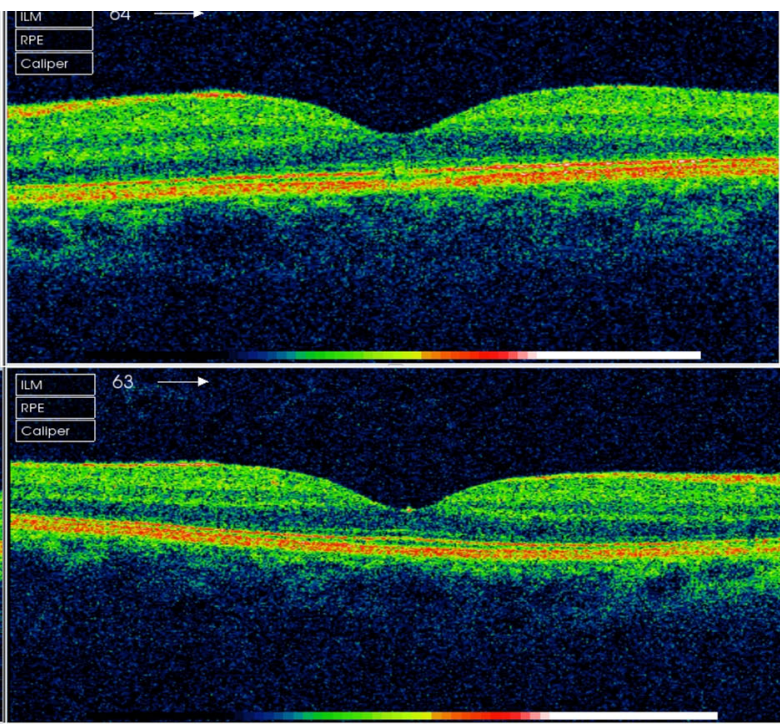

after cessation of sertraline, with resolution of $\mathrm{RPE} /$ retinal changes

\section{Case 3}

A 68-year-old White woman presented with bilateral blurred vision and a VA of 20/30 OD and 20/40 OS. Dilated fundus examination revealed pre-existing mild non-proliferative diabetic retinopathy. There were new bilateral central macular changes with OCT imaging (Fig. 3) demonstrating bilateral subfoveal disruption affecting the outer retinal layers and retinal pigment epithelium (RPE), worse on the left. Anterior segment examination was unremarkable and intraocular pressures were normal. OCT angiography showed no notable abnormality of retinal vasculature and traditional and blue-wave autofluorescence 


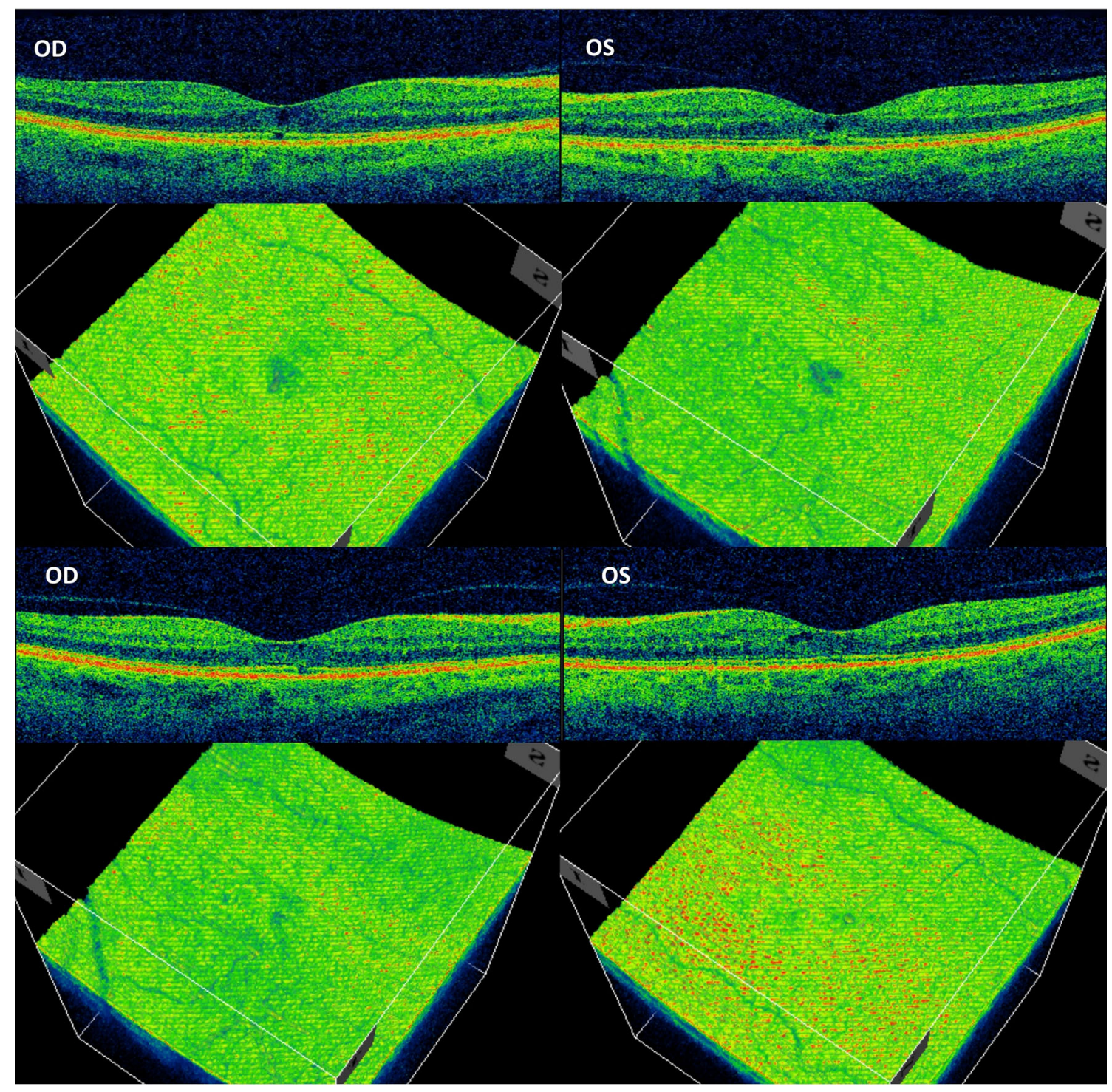

Fig. 3 SD-OCT imaging in cross-sectional and en face orientation. The top half shows imaging from initial presentation; note the lobulated "three-leaf clover" appearance of the maculopathy en face SD-OCT imaging.

imaging. This was conducted using the Spectralis platform (Heidelberg Engineering, Heidelberg, Germany) and did not support alternative diagnoses such as macular telangiectasia type 2 .

The patient had previously taken other SSRIs including fluoxetine and citalopram over a 7 -year period, with no visual symptoms. Sertraline had been initiated for depression
Imaging shown in the bottom half 14 months postsertraline cessation shows improvement in the outer retinal/RPE layers bilaterally with some residual maculopathy

20 months before presentation. Other medical history included well-controlled diabetes type II, hypertension, hypothyroidism, osteoarthritis and gastric bypass surgery. Other regular medications included metformin, losartan, levothyroxine, lansoprazole and aspirin.

VA further declined to $20 / 30$ OD and 20/80 OS before sertraline cessation. Nine months after sertraline cessation, VA improved to 20/25 
OD and 20/30 OS, and follow-up OCT (Fig. 3) demonstrated resolution of subfoveal disruption in the left eye and improvement of the disruption in the right eye.

\section{DISCUSSION}

To date the ocular side-effect profile of sertraline has been limited, with most published reports describing intraocular pressure changes and optic neuropathy [11]. In this series, we highlight three cases of presumed sertraline-induced maculopathy, to add to the five existing published case reports at the time of writing. In our cohort we noted a wide range of sertraline exposure time before developing symptoms, ranging from 4 weeks to over 5 years. Similarly, after sertraline cessation there was variation in final BCVA and degree of maculopathy resolution, perhaps relating to duration of sertraline exposure.

In our cohort, it appeared that shorter sertraline exposure times resulted in the best reversal of symptoms. The shortest exposure time of 4 weeks resulted in rapid resolution of VA and OCT-scan subfoveal abnormalities on sertraline cessation. The longer 20-month exposure time led to satisfactory improvement in VA from 20/80 OS to 20/30 OS, however with residual OCT abnormalities. The exposure time of 5 years resulted in no visual acuity improvement despite architectural improvement on OCT scan. This appears to be in keeping with Agarwal et al.'s case of presumed sertraline-associated maculopathy following 7 months of sertraline exposure, in which VA improved after 3 months cessation from 20/60 OD and 20/40 OS, to 20/30 bilaterally. Mason et al. and Sener et al. described longer exposure periods of 1 year and over 1 year, respectively, and found no BCVA improvement after treatment cessation.

Anatomical changes found on OCT in our cases were consistent with each other, demonstrating RPE and outer retinal layer disruption. None of the patients gave a history of similar causes of such problems, such as exposure to amyl nitrate or history of solar/laser trauma [12]. Cases in the literature have more variance, though always involving the RPE. Sener et al.,
Mason et al. and Ewe et al. all noted RPE atrophy, with the latter two specifically noting bull's-eye maculopathy patterns, resembling chloroquine toxicity. Godara et al. noted macular pigment irregularity with small drusen, inner-outer segment layer disruption and thinning of the outer nuclear layer. Agarwal et al. was the only case to find significant cystic changes with elevation of the RPE.

There are several postulated mechanisms for sertraline-associated maculopathy. Ewe et al. hypothesised that the increased bioavailability of serotonin increases activation of phospholipase $C$ via the 5HT2A receptor [13]. This may lead to increased production of intracellular reactive oxygen species and, finally, retinal degeneration. Agarwal et al. suggested that increased levels of serotonin in the central and peripheral nervous system may interact adversely with RPE and photoreceptor serotonin receptors, via a secondary messenger system consisting of cyclic adenosine monophosphate (cAMP) [14]. This may promote ganglion cell apoptosis and maculopathy development [15]. Ultimately, more research is needed on the serotonin receptor profile and activity of the RPE before a satisfactory pathogenic mechanism can be established. Given the low prevalence of macular toxicity associated with sertraline use, there may need to be another predisposing factor required for disease to manifest.

At present, we must be cautious in presuming that all maculopathy cases reported in the literature were definitely caused by sertraline. However, with increasing reports demonstrating a temporal sequence of events and potential improvement on treatment cessation, as well as a deepening understanding of the biological mechanisms, it is becoming increasingly biologically plausible. Clinicians should be aware of this rare, yet potentially reversible sightthreatening complication of sertraline use. 


\section{ACKNOWLEDGEMENTS}

We thank the participants in this case series in helping us highlight this potentially sightthreatening adverse effect.

Funding. The authors declare no potential conflicts of interest, financial or otherwise. No funding or sponsorship was received for the publication of this article.

Authorship. All named authors meet the International Committee of Medical Journal Editors (ICMJE) criteria for authorship for this article, take responsibility for the integrity of the work as a whole, and have given their approval for this version to be published.

Disclosures. Hedayat Javidi, Sarah Ah-Moye, Charles Hennings, Hemal Mehta and Sajjad Mahmood declare they have nothing to disclose.

Compliance with Ethics Guidelines. Written informed consent for publication was obtained in two out of three cases. In the third case, the patient is not identifiable from the available information and has been included due to the rarity of this disease presentation.

Data Availability. Data sharing is not applicable to this article as no datasets were generated or analyzed during the current study.

Open Access. This article is licensed under a Creative Commons Attribution-NonCommercial 4.0 International License, which permits any non-commercial use, sharing, adaptation, distribution and reproduction in any medium or format, as long as you give appropriate credit to the original author(s) and the source, provide a link to the Creative Commons licence, and indicate if changes were made. The images or other third party material in this article are included in the article's Creative Commons licence, unless indicated otherwise in a credit line to the material. If material is not included in the article's Creative Commons licence and your intended use is not permitted by statutory regulation or exceeds the permitted use, you will need to obtain permission directly from the copyright holder. To view a copy of this licence, visit http://creativecommons.org/licenses/by$\mathrm{nc} / 4.0 /$.

\section{REFERENCES}

1. Vaswani M, Linda FK, Ramesh S. Role of selective serotonin reuptake inhibitors in psychiatric disorders: a comprehensive review. Prog Neuro-Psychopharmacol Biol Psychiatry. 2003;27(1):85-102.

2. Prescribing and Medicines Team. 2017. Prescriptions Dispensed in the Community, England 2006 to 2016 [Internet]. Leeds: NHS Digital; [cited 2019 May 19]. Available from: https://files.digital.nhs.uk/ publication/s/o/pres-disp-com-eng-2006-16-rep. pdf. 13 May 2019

3. van Harten J. Clinical pharmacokinetics of selective serotonin reuptake inhibitors. Clin Pharmacokinet. 1993;24(3):203-20.

4. Ho HY, Kam KW, Young AL, et al. Acute angle closure glaucoma after sertraline. Gen Hosp Psychiatry. 2013;35(5):5751-6572.

5. Lehman NL, Johnson LN. Toxic optic neuropathy after concomitant use of melatonin, zoloft, and a high-protein diet. J Neuroophthalmol. 1999;19(4): 232-4.

6. Mason JO 3rd, Patel SA. Bull's eye maculopathy in a patient taking sertraline. Retin Cases Brief Rep. 2015;9(2):131-3.

7. Ewe SY, Abell RG, Vote BJ. Bilateral maculopathy associated with sertraline. Australas Psychiatry. 2014;22(6):573-5.

8. Sener EC, Kiratli H. Presumed sertraline maculopathy. Acta Ophthalmol Scand. 2001;79(4):428-30.

9. Godara P, Rha J, Tait D, et al. Unusual adaptive optics findings in a patient with bilateral maculopathy. Arch Ophthalmol. 2010;128(2):253-4.

10. Agarwal A, Aggarwal K, Kumar A, et al. Bilateral cystoid macular edema misdiagnosed as pars planitis in a patient on sertraline therapy. Am J Ophthalmol Case Rep. 2018;11:135-8.

11. Costagliola C, Parmeggiani F, Semeraro F, et al. Selective serotonin reuptake inhibitors: a review of its effects on intraocular pressure. Curr Neuropharmacol. 2008;6(4):293-310. 
12. Robertson DM, Lim TH, Salomao DR, et al. Laser pointers and the human eye: a clinicopathologic study. Arch Ophthal. 2000;118(12):1687-91.

13. Nash M, Flanigan T, Leslie R, Osborne N. Serotonin$2 \mathrm{~A}$ receptor mRNA expression in rat retinal pigment epithelial cells. Ophthalmic Res. 1999;31(1):1-4.

14. Pootanakit $\mathrm{K}$, Brunken WJ. Identification of 5-HT(3A) and 5-HT(3B) receptor sub- units in mammalian retinae: potential pre-synaptic modulators of photoreceptors. Brain Res. 2001;896(1-2): 77-85.

15. Nash MS, Wood JP, Osborne NN. Protein kinase C activation by serotonin potentiates agonist-induced stimulation of cAMP production in cultured rat retinal pigment epithelial cells. Exp Eye Res. 1997;64(2):249-55. 